\title{
Agreement between Arthroscopy and Saline Magnetic Resonance Shoulder Arthrography in Adolescent Patients - Evaluation of Location and Extent of Injury of Labral Tears
}

\author{
Michael Fadell, MD ${ }^{1,2^{*}}$, David Howell,3, Jill Stein ${ }^{1,2}$, Marty McGraw ${ }^{1,2}$, Alexia Gagliardi ${ }^{3}$, Stephanie \\ Pearce $^{3,4}$ and Jay Albright ${ }^{3,4}$
}

${ }^{1}$ Department of Radiology, Children's Hospital Colorado, USA

${ }^{2}$ Department of Radiology, University of Colorado School of Medicine, USA

${ }^{3}$ Sports Medicine Center, Children's Hospital Colorado, USA

${ }^{4}$ Department of Orthopedics, University of Colorado School of Medicine, USA

*Corresponding author: Michael Fadell II, MD, Lucile Packard Children's Hospital at Stanford, Clinical Associate Professor of Radiology, Stanford University School of Medicine, 725 Welch Road - Room G780, Stanford, CA 94304-5913, USA, Tel: (650)-736-0458, Fax: (650)-724-2521

\begin{abstract}
Objective: To compare potential differences in size and extent of labral tears on magnetic resonance arthrography of the shoulder performed with saline to shoulder to arthroscopy as the gold standard in adolescent patients with tears of the glenoid labrum.

Materials and methods: Pre-operative saline magnetic resonance arthrograms of the glenoid labrum were assessed by two radiologists for location and extent of the labral tear based on the clock-face method of visualization. The radiology findings were compared to the surgeon's recorded arthroscopic findings (the start and end of the labral tear based on the clock-face method and the total range) in the operative report. One-way analysis of variance was used to compare saline magnetic resonance arthrograms and arthroscopic findings.

Results: Sixteen shoulder saline magnetic resonance arthrograms were included (15 underwent unilateral arthroscopic labral repair, 1 underwent bilateral arthroscopic labral repair. Most patients were male with an average of 17 years of age (range: 15-19 years) at the time of surgery. On average, 54 days elapsed between pre-operative imaging and surgery (range: 9-204 days). Both raters agreed that $100 \%$ of the examinations were of diagnostic quality. There were no significant differences between surgical report and the magnetic resonance arthrography raters for the mean
\end{abstract}

clock face start $(p=0.47)$, end $(p=0.67)$, or total range of the tear $(p=0.97)$. The mean range of the tear was 4 hours for all three raters.

Conclusion: Saline contrast for magnetic resonance shoulder arthrography in adolescent patients is a reliable imaging technique when evaluating the glenoid labrum for the location and extent of injury and is a prudent alternative to gadolinium based contrast agents in adolescent patients.

\section{Keywords}

Arthrography, Arthroscopy, Shoulder, MRI, Adolescent, Saline

\section{Introduction}

Magnetic resonance arthrography (MRA) is often used over conventional MRI in the adolescent population for the evaluation of intra-articular cartilaginous and fibrocartilaginous injuries of the shoulder, as well as injuries to tendons and ligaments. Current MRA techniques use dilute gadolinium-based contrast agents (GBCAs), such as gadopentetate dimeglumine or gadoteridol, as gadolinium is inherently hyperintense on T1 weighted sequences due to resultant shortening of longitudinal regrowth [1]. Gadolinium-based MRA al-

Citation: Fadell M, Howell D, Stein J, McGraw M, Gagliardi A, et al. (2021) Agreement between Arthroscopy and Saline Magnetic Resonance Shoulder Arthrography in Adolescent Patients - Evaluation of Location and Extent of Injury of Labral Tears. Int J Radiol Imaging Technol 7:075. doi.org/10.23937/25723235.1510075

Accepted: April 06, 2021; Published: April 08, 2021

Copyright: (C) 2021 Fadell M, et al. This is an open-access article distributed under the terms of the Creative Commons Attribution License, which permits unrestricted use, distribution, and reproduction in any medium, provided the original author and source are credited 
Table 1: Specific sequences of the saline MRA protocol along with the scan time for each sequence.

\begin{tabular}{|c|c|c|c|}
\hline Sequence & $\begin{array}{l}\text { Repetition time (ms) } \\
\text { (TR) }\end{array}$ & Echo time (ms) (TE) & $\begin{array}{l}\text { Time to complete } \\
\text { (MM:SS) }\end{array}$ \\
\hline Axial T2 with fat time satuartion & 3500 & 60 & $3: 12$ \\
\hline Coronal T1 & 850 & 8 & $2: 53$ \\
\hline Coronal T2 with fat satuaration & 3500 & 60 & $2: 55$ \\
\hline Saggital T2 with fat satuartion & 3500 & 60 & 3:16 \\
\hline $\begin{array}{l}\text { Abduction and external rotation } \\
\text { with fat saturation }\end{array}$ & 3300 & 50 & $3: 42$ \\
\hline
\end{tabular}

Slice thickness $3 \mathrm{~mm}$; Inter slice gap $0.3 \mathrm{~mm}$; FOV $13 \mathrm{~cm}$; Matrix $300 \times 432$

most invariably uses fat saturated (FS) MR spin echo T1 weighted sequences, which allows for excellent contrast between the T1 bright intra-articular gadolinium and the lower signal intensity labrum and soft tissues. Normal saline (hereafter, saline) is inherently hyperintense on $\mathrm{T} 2$ weighted sequences due to a combination of high hydrogen ion content as well as F. Because of this, saline MRA (SaMRA), when used in conjunction with T2 FS sequences, also offers excellent contrast between the 22 bright intra-articular contrast and the lower signal intensity labrum and soft tissues. Saline is also a potentially safer alternative given rare adverse events and incomplete clearance with GBCAs [2,3].

The use of saline contrast for shoulder MRA has been shown to demonstrate comparable sensitivity and specificity in the evaluation of joint pathology in the adult population when compared with traditional gadolinium-based MRA [4,5], but evidence in adolescent patients is currently absent. Accordingly, the purpose of this investigation was to examine differences in location and extent of injury between saline contrast for shoulder MRA and surgery among a cohort of adolescent patients who underwent arthroscopic surgery for tears of the glenoid labrum based on SaMRA.

\section{Methods}

This was an observational and retrospective study approved by the institutional review board. We began exclusively utilizing SaMRA at our institution in the fall of 2016. Between 2016 and 2019 a total of eighty two patients underwent SaMRA. Twenty-one of these patients underwent SaMRA prior to same side shoulder arthroscopy during this time period and were initially included in the study. Patients that did not undergo subsequent arthroscopy following SaMRA were not included in the study as the objective of the study was to compare specific parameters, namely location and extent of injury. The only exclusion criteria were prior shoulder surgery, which led to the exclusion of two patients. This resulted in the inclusion of a total of sixteen cases of glenoid labral tears. All patients included in the study underwent standard arthrographic injection of iodinated contrast under fluoroscopic guidance via a posterior approach prior to SaMRA imaging. Arthrography was performed with a $5 \mathrm{~cm}$ 21-gauge needle after local fluoroscopic anesthesia was induced with $1 \%$ Lidocaine. The arthrographic solution was a 1:2 dilution of Isovue 300 (iopamidol lopamiro 200; Bracco Diagnostics, Milan, Italy) with normal saline and the volume injected varied between 10-15 ML. SaMRA included the following sequences: Axial T2 FS, Coronal T1, Coronal T2 FS, Sagittal T2 FS, and when tolerated by the patient, ABER (abduction with external rotation) T2 FS (Table 1).

The SaMRAs were independently reviewed, in random order to avoid bias, by two board certified pediatric radiologists with 5 and 12 years of experience. Readers were instructed to assess the location and extent of labral injury, were blinded to the arthroscopy surgical report at the time of review and were not involved with the arthrographic injections. The quality of the imaging study was initially scored as diagnostic, sub-optimal, or non-diagnostic. A study was considered diagnostic if the spatial and contrast resolution were such that the trabeculae and cortex were sharply defined, there was good definition of surrounding tissues, and there was good discrimination of rotator cuff tendons, biceps tendon, labrum, and cartilage from joint fluid. A study was considered suboptimal if the above-mentioned structures were blurred or not sharply defined and a study was considered nondiagnostic if the above-mentioned structures were obscured secondary to motion or other artifact. The extra-articular soft tissues about the rotator cuff were also assessed and rated as either normal or abnormal (abnormal T2 hyperintensity), as a full thickness rotator cuff tear with extravasation of intra-articular saline could manifest as increased signal intensity in the peri-articular soft tissues on fluid sensitive sequences. The SaMRA raters independently assessed the location and extent of the injury to the hour based on the clock-face method of visualizing of the glenoid labrum. A single orthopedic surgeon, with 15 years of experience, performed all the shoulder arthroscopies. The orthopedic surgeon assessed the location and extent of labral injury during arthroscopy using the same methodology as the radiologists. For reference and aligning with previous work [6], the labral tears were described with the following reference points: Superior (12:00) which is designated by the location of the biceps anchor, anterior (3:00), inferior (6:00), and posterior (9:00). This methodology is used in arthroscopy 
for both preoperative planning and for communicating reliable descriptions of labral pathology in operative reports. Our primary outcome variables from this analysis included the start of the tear, the end of the tear, and the total range of the tear, each recorded based on reference to the clock face.

\section{Statistical Methods}

Continuous variables are presented as means and standard deviations, and categorical variables are presented as the number included and the corresponding percentages. To test for differences between SaMRA and surgical findings of labral injury location designated by the clock-face, we used a one-way analysis of variance (ANOVA), where rater (surgical report, MRA rater 1, MRA rater 2) were the independent variables and the clock face start, clock face end, and range of tear were the dependent variables. All statistical tests were two-sided, statistical significance was defined as $p$ $<0.05$, and Stata version 15.0 was used for all statistical analyses.

\section{Results}

Sixteen shoulder SaMRAs performed without complication, all in patients that underwent subsequent same side shoulder arthroscopy, were included in the study. Fifteen of these patients underwent unilateral arthroscopic labral repair and one patient underwent bilateral arthroscopic labral repair as well as pre-operative bilateral SaMRAs. Most patients were male with an average of 17 years of age (range: $15-19$ years) at the time of surgery (Table 2). On average, 54.39 days elapsed between pre-operative imaging and operative

Table 2: Description of the study cohort. Data are presented as means (sd) or number (\% of the study sample).

\begin{tabular}{|l|l|}
\hline Characteristic & Mean (sd) or $\mathbf{n}(\%)$ \\
\hline Age (years) & $17.1(1.2)$ \\
\hline Sex (female) & $3(19 \%)$ \\
\hline $\begin{array}{l}\text { Post-Operative Diagnosis (per } \\
\text { arthroscopy) }\end{array}$ & $16(100 \%)$ \\
\hline
\end{tabular}

management (range: 9-204 days). Both SaMRA raters agreed that $100 \%$ of the studies were diagnostic (Table 3). Two of the SaMRA's were identified as having abnormal peri-articular 72 high signal (Table 3). Upon further review, both raters agreed by consensus that the two cases had the same finding of periarticular signal located adjacent to the axillary pouch, without any features to suggest rotator cuff pathology. There were no significant differences between the surgical report and the SaMRA raters (two Radiologist and the single orthopedic surgeon) for the mean clock face start, end, or total range of labral tears throughout the patient cohort (Table 4). Figure 1 demonstrates a posterior inferior labral tear seen on both arthroscopy (A) and SaMRA (B). Of note, the mean range was similar across all three raters, averaging approximately 4 "hours" on the clock face. Figure 2 shows an example of a glenolabral articular disruption (GLAD lesion) on both SaMRA and arthroscopy.

\section{Discussion}

Our findings indicate that there is no significant difference between surgical findings and SaMRA raters for the mean clock face start/end position or mean total lesion range.

Numerous studies in adults have evaluated the use of saline as an alternative contrast agent for MR shoulder arthrography $[4,5,7,8]$. Helms and colleagues evaluated intra-articular gadolinium versus saline alone in magnetic resonance arthrography (MRA) of the shoulder in patients with a mean age of 36 , concluding that SaMRA is equivalent diagnostically relative to MRA with gadolinium, although the imaging characteristics of SaMRA were simulated by analyzing $T 2$ weighted images in isolation [4]. Separate studies examining shoulder MRA performed with saline showed reliable results in identifying abnormalities of the glenohumeral joint, although the studies did not directly compare saline to intra-articular gadolinium $[7,8]$. Binkert, et al. compared shoulder MRA performed with gadolinium versus Ringer solution to arthroscopy, though also with an older cohort of patients with a mean age 61 , and reported

Table 3: MRI image characteristics for the study cohort.

\begin{tabular}{|l|l|l|l|}
\hline Image Characteristic & MRA rater 1 & MRA rater 1 \\
\hline \multirow{3}{*}{ Image quality of the study } & Diagnostic study & $16(100 \%)$ & $16(100 \%)$ \\
\cline { 2 - 3 } & Sub-optimal study & $0(0 \%)$ & $0(0 \%)$ \\
\cline { 2 - 3 } & Non-diagnostic study & $0(0 \%)$ & $0(0 \%)$ \\
\hline \multirow{2}{*}{$\begin{array}{l}\text { Extra-articular soft tissues } \\
\text { about the rotator cuff? }\end{array}$} & Normal & $16(100 \%)$ & $14(87 \%)$ \\
\cline { 2 - 4 } & Abnormal T2 high signal & $0(0 \%)$ & $2(13 \%)$ \\
\hline
\end{tabular}

Table 4: Mean (SD) values for clock face start, end, and range for labral tears as rated by the surgical report and two MRA raters.

\begin{tabular}{|l|l|l|l|l|}
\hline Rater & Surgical report & MRA rater 1 & MRA rater 2 & P value \\
\hline Clock face start & $3: 37(2: 13)$ & $5: 03(4: 33)$ & $3: 45(3: 30)$ & 0.46 \\
\hline Clock face end & $6: 50(1: 08)$ & $7: 07(2: 23)$ & $7: 30(2: 23)$ & 0.67 \\
\hline Clock face range & $3: 54(1: 05)$ & $3: 52(2: 03)$ & $3: 45(2: 14)$ & 0.97 \\
\hline
\end{tabular}




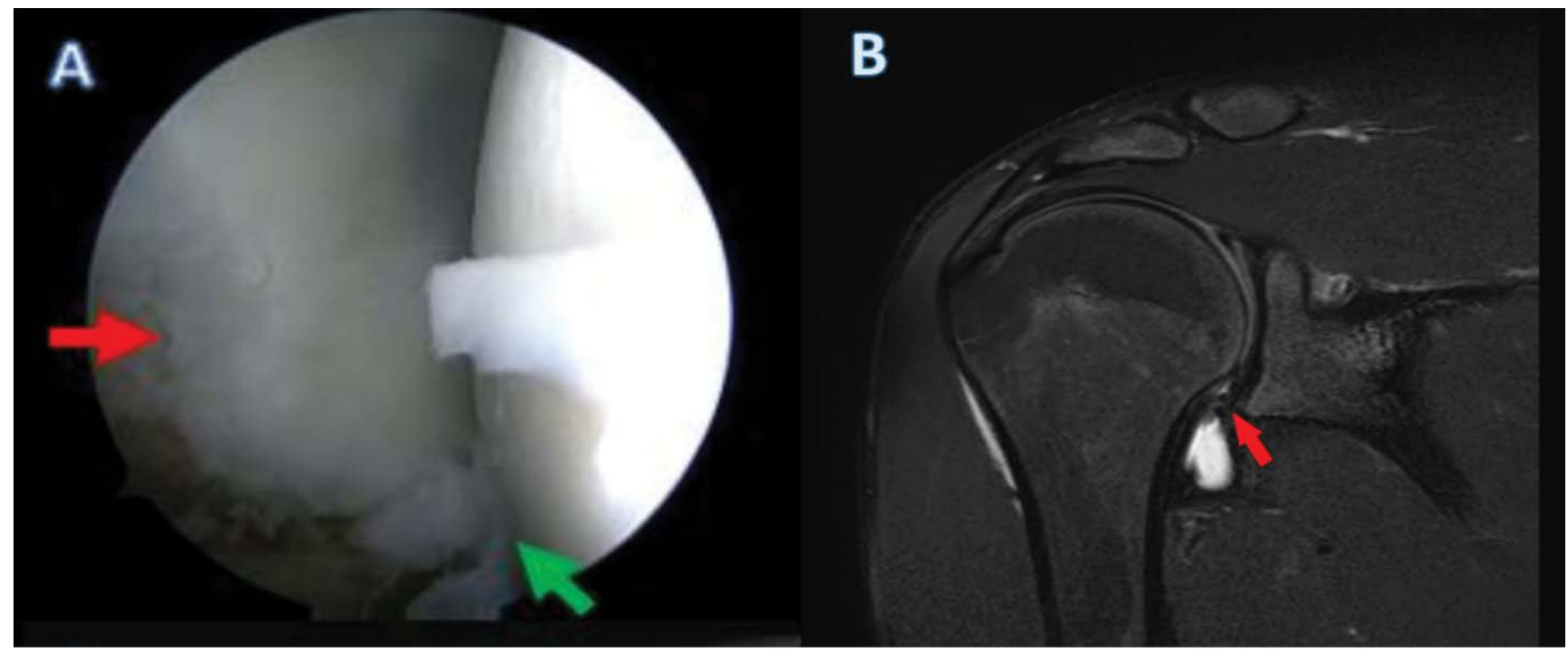

Figure 1: Arthroscopic (A) and SaMRA images $(B)$ of the glenohumeral joint in a 17-year-old male. Full thickness labral tear of the inferior and posterior labrum extending from the 4 (green arrow in A) to 8 o'clock (red arrow in A and B). Diminished size of the inferior labrum along with irregularity on SaMRA (red arrow in B). A small amount of fluid is also incidentally seen within the subdeltoid bursa.
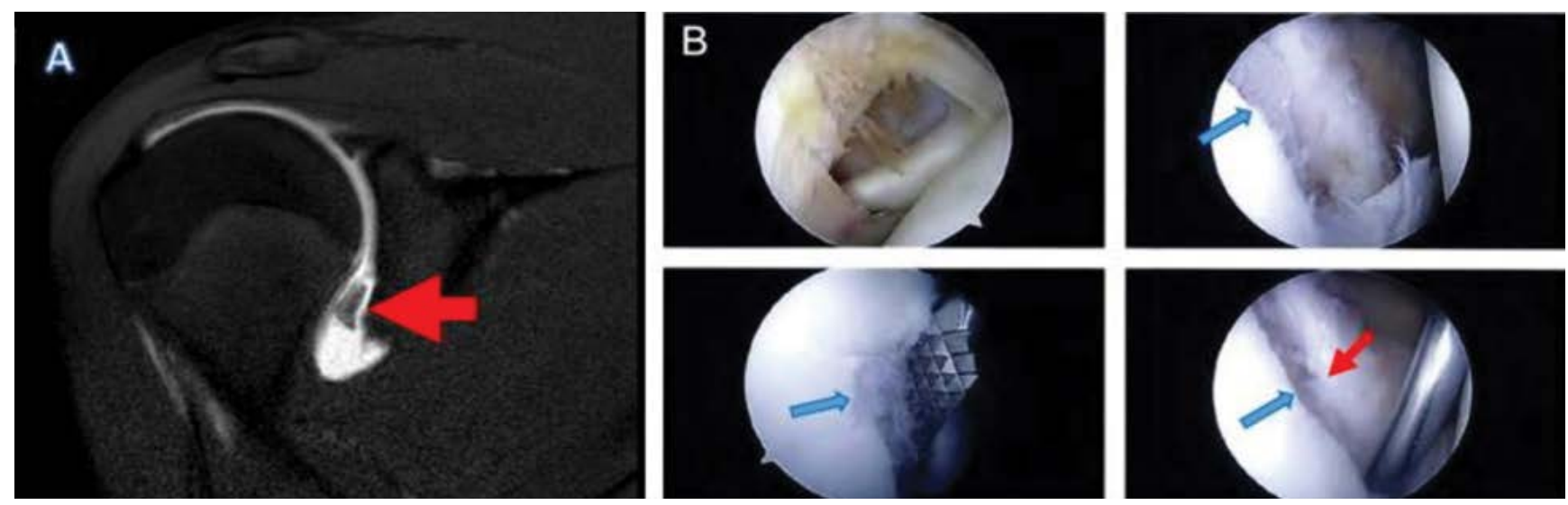

Figure 2: Coronal SaMRA (A) and arthroscopic (B) images of the right glenohumeral joint in a 17-year-old male demonstrate an anterior inferior glenolabral articular disruption (GLAD) (red arrow in A). This can be seen from the 2 to 6 o'clock positions (red arrows in B). Corresponding injury to the articular cartilage is also well seen (blue arrows in $B$ ).

equivalent diagnostic accuracy between methods [5]. Most recently, Singer and colleagues compared the diagnostic performance of SaMRA to Gadolinium-based MRA for the detection of labral and rotator cuff injuries with average ages of 35 and 51 respectively, again showing comparable diagnostic performance [9].

Though GBCAs are in common use today and generally considered innocuous, rare adverse events may occur, such as allergic reactions and nephrogenic systemic fibrosis (a disease of fibrosis caused by gadolinium exposure in patients with renal insufficiency) [10]. More recent work has demonstrated the incomplete clearance of GBCAs from the body [11-13]. Regulatory agencies including the American College of Radiology, American Society of Neuroradiology as well as the FDA and the European Medicine Agencies have all encouraged physicians to use GBCAs only when clinically necessary $[2,3]$.

In addition to being a potentially safer option, some cost savings along with shorter overall scan time are some additional benefits to SaMRA. The estimated cost savings at our institution based on performing a total of 90 shoulder MRAs annually along with the institutional cost for a single dose of Multi-Hance ( $\$ 2$ per $\mathrm{mL}$ for a 10 $\mathrm{mL}$ bottle) is approximately $\$ 1,800$ per year. Given that the estimated decrease in scan time, due to completely eliminating the T1 FS sequences typically included in Gadolinium-based MRA protocols, for an adolescent patient undergoing a shoulder MRA (including repositioning for an ABER sequence) is approximately 10 minutes, this translates to an estimated scan time savings of 15 hours annually at our institution.

Despite the potential of SaMRA as an alternative, there are several limitations associated with this technique in the shoulder. Differentiating extravasation through a full thickness rotator cuff tear from a bursal fluid collection is a potential difficulty given that saline is generally isointense to bursal fluid (either subdeltoid 
or subacromial) on T2 weighted sequences. In the adolescent population, however, full thickness rotator cuff pathology is rare, and is typically accompanied by a gap in the tendon [14], likely easing this limitation to some degree. It should also be noted that, SaMRa may lead to diagnostic difficulty when arthrographic injection is performed via the rotator cuff interval guidance.

In addition to the possible challenges related to the saline technique, our study had additional limitations. Our sample was small and not random, as each patient underwent arthroscopic glenoid labral repair. Our study sample also did not include a control group without labral injury, as operative assessment would not have been performed. Intrinsic selection bias was likely present for the raters, though they were not informed that all cases were positive. We also did not control for the time interval between MRI to arthroscopy, which ranged from just over a week to just over seven months. The abnormal labrum may have undergone some change during this time, limiting the accuracy of MRA.

Though GBCAs are diluted in MRA and are administered intraarticularly and there is a low likelihood for repeat examinations, transitioning to shoulder SaMRAs in adolescent patients is still likely a prudent alternative [15].

\section{Conclusion}

Our study adds to body of literature confirming that saline is a safe, accurate, and readily available alternative to GBCAs for MR shoulder arthrography. However, unlike the previous studies, our cohort was adolescent, the age group ideally suited for saline arthrography given the possibility of greater cumulative exposure to GBCA's throughout one's life.

\section{Compliance with Ethical Standards}

This study received approval from the local institutional review board before commencement.

\section{Funding}

This study was unfunded.

\section{Conflicts of Interest}

The authors declare no conflicts of interest related to this study. Unrelated to this study, Dr. Howell has received research support from the Eunice Kennedy Shriver National Institute of Child Health \& Human Development, the National Institute of Neurological Disorders And Stroke, and from a research contract between Boston Children's Hospital, Cincinnati Children's Hospital Medical Center, and EIMindA Ltd. Dr. Albright has received research support from Pediatric Orthopedic Society of North America (POSNA) and from Arthrex Inc. He has also received honoraria from Arthrex for speaking about surgical outcomes. The other authors declare no potential conflicts of interest.

\section{References}

1. Xiao YD, Paudel R, Liu J, Ma C, Zhang ZS, et al. (2016) MRI contrast agents: Classification and application (Review). Int J Mol Med 38: 1319-1326.

2. (2017) FDA Medical Imaging Drugs Advisory Committee.

3. (2017) European medicines agency final opinion on use of linear gadolinium agents in body scans.

4. Helms CA, McGonegle SJ, Vinson EN, Whiteside MB (2011) Magnetic resonance arthrography of the shoulder: Accuracy of gadolinium versus saline for rotator cuff and labral pathology. Skeletal Radiol 40: 197-203.

5. Binkert CA, Zanetti M, Gerber C, Hodler J (2001) MR arthrography of the glenohumeral joint: Two concentrations of gadoteridol versus ringer solution as the intraarticular contrast material. Radiology 220: 219-224.

6. Hansen $\mathrm{CH}$, Asturias AM, Pennock AT, Edmonds EW (2020) Adolescent posterior-superior glenoid labral pathology: Does involvement of the biceps anchor make a difference? Am J Sports Med 48: 959-965.

7. Willemsen UF, Wiedemann E, Brunner U, Scheck R, Pfluger T, et al. (1998) Prospective evaluation of MR arthrography performed with high-volume intraarticular saline enhancement in patients with recurrent anterior dislocations of the shoulder. AJR Am J Roentgenol 170: 79-84.

8. Tirman PF, Stauffer AE, Crues JV, Turner RM, Nottage WM, et al. (1993) Saline magnetic resonance arthrography in the evaluation of glenohumeral instability. Arthroscopy 9: 550-559.

9. Singer $A D$, Rosenthal J, Umpierrez M, Guo Y, Gonzalez F, et al. (2020) A comparison of saline and gadolinium shoulder MR arthrography to arthroscopy. Skeletal Radiol 49: 625-633.

10. Thomsen HS (2009) Nephrogenic systemic fibrosis: History and epidemiology. Radiol Clin North Am 47: 827-831.

11. Kanda T, Osawa M, Oba H, Toyoda K, Kotoku J, et al. (2015) High signal intensity in dentate nucleus on unenhanced t1-weighted mr images: Association with linear versus macrocyclic gadolinium chelate administration. Radiology 275: 803-809.

12. Flood TF, Stence NV, Maloney JA, Mirsky DM (2017) Pediatric brain: Repeated exposure to linear gadolinium-based contrast material is associated with increased signal intensity at unenhanced T1-weighted MR imaging. Radiology 282: $222-228$.

13. Roberts DR, Holden KR (2016) Progressive increase of T1 signal intensity in the dentate nucleus and globus pallidus on unenhanced T1-weighted MR images in the pediatric brain exposed to multiple doses of gadolinium contrast. Brain Dev 38: 331-336.

14. Alley MC, Banerjee S, Papaliodis D, Tsitos K, Zanaros GS (2016) Transosseous physeal-sparing rotator cuff repair in an adolescent football player. Orthopedics 39: e353-e358.

15. Dillman JR, Davenport MS (2020) Gadolinium retention - 5 years later.... Pediatr Radiol 50: 166-167. 